\title{
Intelligence: Exact computation or biofunctional cognition
}

\author{
ANTONY SATYADAS, ASGHAR IRAN-NEJAD, HUI CHUAN CHEN, and BRAD CHISSOM \\ University of Alabama, Tuscaloosa, Alabama
}

\begin{abstract}
What is it that makes human beings so good at fuzzy judgments and so poor at precise computation? This paper first addresses this question in the context of a biofunctional theory of how the nervous system functions and then discusses a fuzzy intelligence evaluation system for determing the intelligence of individuals.
\end{abstract}

Intelligence is commonly used interchangeably with competence, expertise, native talent, cultural talent, personal control, schooling, social interaction, and IQ. Most psychological definitions of intelligence include a knowledge component (roughly crystallized intelligence) and a problem-solving component (fluid intelligence); however, the nature of the relationship between these components is not well understood. One major obstacle toward understanding is that most cognitive theories of knowledge process and store information in discrete chunks, making it difficult to handle the inherently subjective nature of knowledge categories, real-world problem solving, and their relationship.

It is also difficult to see how recent neural computation networks of cognitive science and artificial intelligence can handle fuzzy categories and subjective decision making. Networks represent knowledge categories as discrete units and connection weights. Fuzziness in them represents a departure from idealized clear-cut sets. The formal apparatus of fuzzy set theory, for instance, is of little help to these networks in which "units define clear-cut sets with no marginal cases"' (Hinton, 1989, p. 60). Knowledge categories, such as old (Hinton, 1989), game (Wittgenstein, 1953), and intelligence (Spearman, 1927), on the other hand, are inherently fuzzy and do not readily lend themselves to clean-cut set definitions. This is probably why attempts at discrete-construct definitions have often resulted in the conclusion that terms such as intelligence have so many meanings that they finally have none (Spearman, 1927).

What is it that makes human beings so good at fuzzy judgments and so poor at precise computation? We cannot judge (compute) exactly how old, how tall, or how heavy someone is, but we are very good at making realistic estimates. This is the opposite of what exact computation theories would suggest. Problems associated with assigning discrete units and connection weights to attributes such as old are often recognized (Hinton, 1989); however, the severity of these problems for related theories and their implications for the way we think about human

Correspondence should be addressed to A. Iran-Nejad, Area of Behavioral Studies, University of Alabama, Box 870231, Tuscaloosa, AL 35487. intelligence are very difficult to expose. For example, if we could partition all people into two clear-cut sets, a separate network containing discrete units might be possible for each set. Unfortunately, such partitioning would be meaningless in any realistic sense because there are always contexts in which the term old is applicable to all people ranging from less than a few hours to more than 100 years old. This takes us back to the inherently fuzzy nature of human knowledge. Recent developments in biofunctional cognition (Iran-Nejad, Marsh, \& Clements, 1992; Iran-Nejad \& Ortony, 1984; Marsh \& Iran-Nejad, 1992) suggest that there may exist a solution to this dilemma.

Biofunctional theory implies that the brain first creates a nondiscrete type of knowledge called thematic knowledge and uses it to create momentary knowledge categories in particular contexts. The thematic knowledge that the brain creates is nondiscrete in much the same sense, by analogy, that the sunshine the sun creates is nondiscrete. It is exactly in this sense, according to biofunctional theory, that human knowledge is inherently fuzzy and unanalyzable. Trying to discretize it into units and connections is analogous to trying to build sunshine balls and ropes out of sunshine.

According to biofunctional theory, the brain engages in two qualitatively different kinds of activity to create thematic or categorical knowledge. Thematic knowledge is created when brain subsystems (e.g., the visual or affective subsystems) stay on continuously just as, by analogy, the lights in a room can stay on continuously. Categorical knowledge, on the other hand, is created when constellations of brain microsystems, whose elements are distributed across different subsystems, fire blinks in the manner of the blinking traffic arrow on the freeway. In this fashion, ongoing brain activity (OBA) or thematic knowledge serves as the context for momentary constellation firing (MCF) or categorical knowledge. This means that human knowledge is inherently subjective, relative, and imprecise. Thematic knowledge cannot be partitioned into discrete units or sets of units at all because it is inherently unanalyzable. Categorical knowledge is the only unit-level knowledge that the brain creates, but it is inherently momentary and relative (i.e., theme bound or context bound). 
If the biofunctional approach is correct, the two different kinds of brain activity must constitute two major sources of variation in intellectual functioning. In addition, a third major source of intellectual variation is the capacity of the nervous system to engage in biofunctional self-regulation (BSR). The purpose of this paper is to explore the extent to which these major sources lend themselves to formal analysis in terms of fuzzy mathematics in which vague conceptual phenomena can be precisely and rigorously studied under a well-developed mathematical framework. More specifically, we propose a fuzzy intelligence evaluation expert system that can use these three sources of data from individuals to evaluate their intelligence. It is hypothesized, for instance, that the three sources precipitate differentially in the form of linguistic realizations. Thus, terms such as sharp, bright, and methodical refer to fuzzy variables that cognitively represent the brain's MCF, OBA, and BSR functioning, respectively.

\section{Fuzzy Sets}

A fuzzy set contains a class of elements in which no sharp, all-or-none ( 0 or 1$)$ boundaries separate the elements that belong to the set. The elements of the set have various degrees (from 0 to 1 ) of membership that indicate the grade of these elements in the set. Larger values denote higher degrees of membership. A mathematical function that assigns values to these elements is called a membership function. The theory of fuzzy sets represents a conceptual framework for a systematic treatment, in both quantitative and qualitative form, of the vagueness and uncertainty inherent in human knowledge and evaluation processes. Vagueness or fuzziness comes from the continuous nature of human thematic knowledge, which permits no sharp boundaries, and the relative nature of human categorical knowledge, which allows no absolute attributes. Uncertainty, on the other hand, seems to arise from incompleteness and contributes to the degree of confidence in an evaluation. Consider, for instance, the attribute height (or tallness). Figure 1a is a way of representing height of X thematically. The horizontal axis represents height of $X$, and the vertical axis represents its degree of membership in the fuzzy set. The figure may be viewed as a good external representation of thematic human knowledge about tallness, because its two axes represent continuous variation and the figure as a whole constitutes a nondiscrete space. Moreover, the set of individual membership curves provides a suitable way of drawing fuzzy categorical inferences from this thematic knowledge base. Note that, at the most general level, all of the members of the set of qualifier curves shown in Figure 1a apply, in principle, continuously to all the points on the referential set (horizontal axis). In other words, for all Xs, it can be said that $\mathrm{X}$ is not tall, $\mathrm{X}$ is somewhat tall, $\mathrm{X}$ is (prototypically) tall, $\mathrm{X}$ is very tall, or $\mathrm{X}$ is extremely tall.

More specific categorization is achieved by moving the focus of attention to one of the curves and applying it to a particular point in the referential set. For example, "John is somewhat tall" is a fuzzy categorical inference. It is categorical to the extent that it singles out somewhat, as opposed to extremely and so on, for application to particular members of the referential subset-namely, those values of height of $X$ that can be characterized as somewhat, as opposed to extremely, tall. It is fuzzy, to the extent that somewhat belongs to a set whose members apply to the above referential subset of $\mathrm{X}$ in varying degrees. Thus, given height of John as the focal member of the referential subset of $X$ (see Figure 1b), we have: John is not tall (0.61), John is somewhat tall (1.00), John is tall (0.39), John is very tall $(0.21)$, and John is extremely tall $(0.11)$. Here, the number in parentheses refers to the grade of membership of John's height in the fuzzy set tallness. These numbers correspond to a height of $5 \mathrm{ft} 10 \mathrm{in}$. in Figure 1b. In this fashion, the system expresses height of John linguistically as somewhat tall, which captures the principle of minimum specificity inherent in human thought and language. According to this principle, the less specific linguistic value, rather than the exact numerical value, quantifies thoughts and relationships. Figure 1c replaces height of $\mathrm{X}$ with the base set (ranging from 0 to 1). Thus, Figure 1c represents, in principle, all applications of fuzzy categories including intelligence.

Parallel to fuzzy set theory, Zadeh (1965) proposed a fuzzy propositional logic, that allows these grades of membership in a fuzzy set to be interpreted as truth values of a

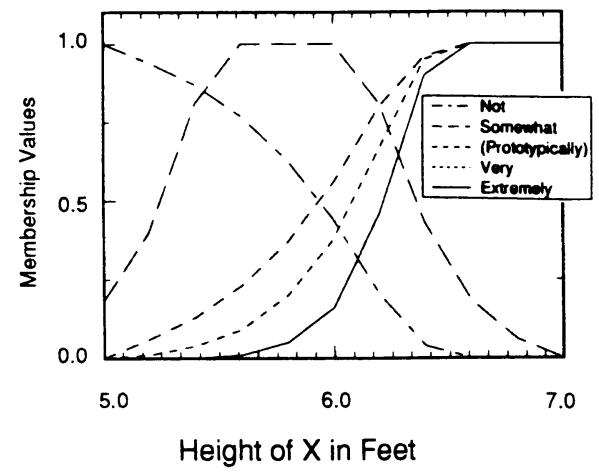

b

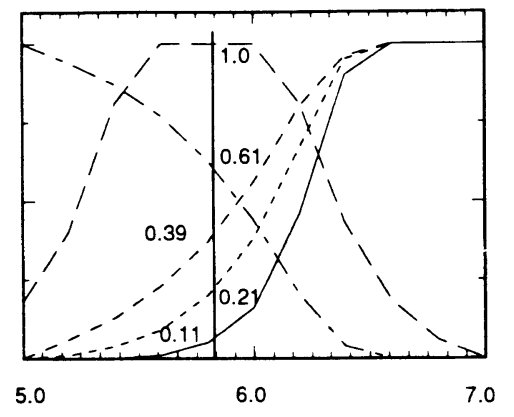

Height of John in Feet c

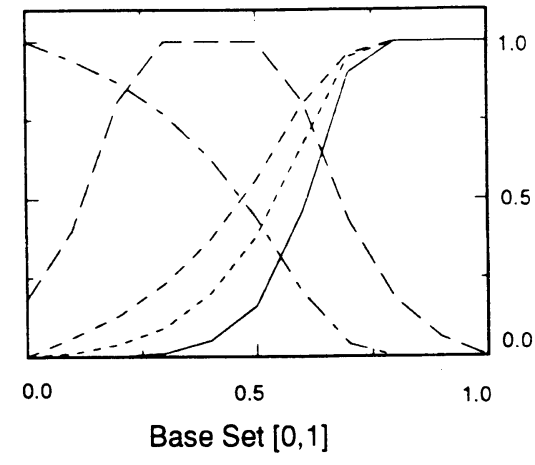

Figure 1. Membership curves for fuzzy qualifiers and (a) height of $X$, (b) height of John, and (c) the standard base set (0,1) as the referential set. As an example, the solid line in Panel b represents membership in different curves for a height of $5 \mathrm{ft} 10$ in. 
a predicate. Thus, the number in parentheses, the truth value of each of the above mentioned propositions, can be represented by the fuzzy set (not true, somewhat true, true, very true, definitely true).

\section{Multicriteria Evaluation}

Let us consider a simple evaluation process such as determining the potential of John as a basketball player. Like height, there could be several other factors or criteria that evaluators might use. Moreover, the evaluators would usually have some heuristics to relate these criteria, which could be stated in the form of if-then rules: "If John is very tall and John is athletic, then John has good potential to be a basketball player." Evaluation rules for intelligence may be expressed similarly. For example, "If $\mathrm{X}$ is bright and $\mathrm{X}$ is sharp and $\mathrm{X}$ is methodical, then $\mathrm{X}$ is intelligent." The variables bright, sharp, and methodical can be represented by fuzzy sets. For example, the evaluation of an individual's brightness can have memberships in the set not bright, somewhat bright, and so on. The same holds good for sharpness and methodicalness. Given the membership values for the variables in a rule, fuzzy conjunction and implication procedures can be used to determine a set of corresponding membership values for intelligence. First, we combine the membership values for all variables in the rule using a fuzzy conjunction operation (Bellman \& Zadeh, 1970). Then, Zadeh's (1977) implication procedure for approximate reasoning is applied to obtain membership values for intelligence. This would represent the evaluation of an individual's intelligence, in terms of degree of membership in the set, from not intelligent to extremely intelligent. There will be one such subset per rule. The evaluations for individual rules are then combined, using a fuzzy aggregation operation, to obtain an overall evaluation set. The overall evaluation set will be in the form of a curve that gives membership values for the degrees of intelligence that range from not intelligent to extremely intelligent. To compare individual differences in intelligence, this overall evaluation set can then be evaluated by using various ranking techniques for fuzzy sets.

\section{An Example}

The objective of the proposed expert system is to evaluate the intelligence of individuals using biofunctional, behavioral, or experiential measures of their OBA, MCF, and BSR or, in other words, of how bright, sharp, or methodical they are. Biofunctional measures may include measures of the brain's electrical activity. Behavioral tests may be similar to existing intelligence tests but designed specifically to measure OBA, MCF, and BSR. Experiential measures might include various subjective tests. The measurements could be in numeric or linguistic form.

Given the raw data for a particular individual presented in Table 1, the membership of each variable (OBA, MCF, BSR) in the various fuzzy sets can be computed using the membership functions given in Table 2 . For example, if we use the average values in row 4 of Table 1 , the membership values will be as shown in Table 3 . Thus, for the average reading of 0.60 for OBA in Table 1, by apply- ing the membership function for not bright in Table 2 , we get the membership value of 0.17 for not bright in Table 3. The values in Table 3 can then be related to the person's intelligence by proper rules. We use the following two rules in this paper:

RULE 1: If OBA is extremely bright and MCF is extremely sharp and BSR is extremely methodic, then the individual is extremely intelligent.

RULE 2: If OBA is not bright and MCF is not sharp and BSR is not methodic, then the individual is not bright.

More rules can be added as required. The membership values for brightness, sharpness, and methodicalness for each rule can be combined to obtain the combination value. Thus, using the fuzzy conjunction procedure proposed by Zadeh (1965), for Rule 1 we get 0.10 (M1), which is the minimum of $0.56,0.25$, and 0.10 ; similarly, for Rule 2 we get 0.17 (M2), which is the minimum of $0.17,0.37$, and 0.53 . Now we have individual evaluations M1 from Rule 1 and M2 from Rule 2. To obtain the subset of intelligence membership values for each rule, the fuzzy implication procedure is applied next on these two values over the base set $(0,1)$ in steps of 0.1 as follows:

$$
\mathrm{H}(\mathrm{w}, \mathrm{i})=\min \{1,[1-\mathrm{Mw}+\mathrm{B}(\mathrm{i})]\}
$$

for $w=1,2$ and $i=0.00,0.10,0.20, \ldots 1.00$, where $\mathrm{H}(\mathrm{w}, \mathrm{i})$ is the intelligence membership value for Rule $w$, corresponding to value $\mathrm{i}$ in the base set. $\mathrm{Mw}$ is the combination (minimum) value for Rule $w$, and $B(i)$ is base value $i$ corresponding to the kind of intelligence given by Rule w. Thus, for Rule $1,(w=1), M 1=0.10$, and given a base value 0.00 (i), we compute $B(0.00)$ using the mem-

Table 1

Sample Experimental Values After

Transformation to the Base Set $(0,1)$

\begin{tabular}{cccc}
\hline Sample & OBA & MCF & BSR \\
\hline 1 & 0.6 & 0.5 & 0.6 \\
2 & 0.6 & 0.5 & 0.2 \\
3 & 0.7 & 0.5 & 0.4 \\
Average & 0.6 & 0.5 & 0.4 \\
\hline
\end{tabular}

Table 2

Membership Functions for the Fuzzy Set Qualifiers

\begin{tabular}{cll}
\hline Item & Fuzzy Set Qualifier & Membership Function \\
\hline i & not & $1-\mathrm{iii}$ \\
ii & somewhat & $1-\mathrm{e}^{-(0.25 /|0.4-x|)^{2.3}}$ \\
iii & (prototypically) & $1-\mathrm{e}^{-(0.5 /|1-x|)^{2 . s}}$ \\
iv & very & iii*iii \\
v & extremely & iii*iii*iii \\
\hline
\end{tabular}

Table 3

Membership Values for the Samples in Table 1

\begin{tabular}{lccccc}
\hline Variable & not & somewhat & (prototypically) & very & extremely \\
\hline OBA & 0.17 & 0.83 & 0.83 & 0.68 & 0.56 \\
MCF & 0.37 & 1.00 & 0.63 & 0.40 & 0.25 \\
BSR & 0.53 & 1.00 & 0.47 & 0.22 & 0.10 \\
\hline
\end{tabular}


Table 4

Evaluation Sets for Rule 1, Rule 2, Minimum of Rules

1 and 2, and Normalized Minimum of Rules 1 and 2

\begin{tabular}{lccccccccccc}
\hline & \multicolumn{10}{c}{ Base Values } \\
\cline { 2 - 12 } & 0.0 & 0.1 & 0.2 & 0.3 & 0.4 & 0.5 & 0.6 & 0.7 & 0.8 & 0.9 & $\sim 1.0$ \\
\hline Rule 1 & .90 & .90 & .91 & .93 & .96 & 1.0 & 1.0 & 1.0 & 1.0 & 1.0 & 1.0 \\
Rule 2 & 1.0 & 1.0 & 1.0 & 1.0 & 1.0 & 1.0 & 1.0 & 1.0 & 1.0 & .93 & .83 \\
Overall & .90 & .90 & .91 & .93 & .96 & 1.0 & 1.0 & 1.0 & 1.0 & .93 & .83 \\
Normalized & .41 & .42 & .46 & .57 & .79 & 1.0 & 1.0 & 1.0 & 1.0 & .59 & 0.0 \\
\hline
\end{tabular}

bership function for extremely intelligent. Thus, we get

$$
\mathrm{B}(0.00)=0.00
$$

and

$\mathrm{H}(1,0.00)=$ minimum $(1,1-0.10+0.00)=0.90$.

Performing this for every rule over every value in the base set, we get a $2 \times 10$ matrix of intelligence membership values (Table 4). Next, we determine the overall evaluation set. For each value in the base set, the conjunction (minimum) of the two intelligence values is selected. This yields the overall intelligence values shown in row 3 of Table 4, which are normalized to yield the values in row 4 . Figure 2 shows the results obtained from Rule 1, Rule 2, and their normalized conjunction.

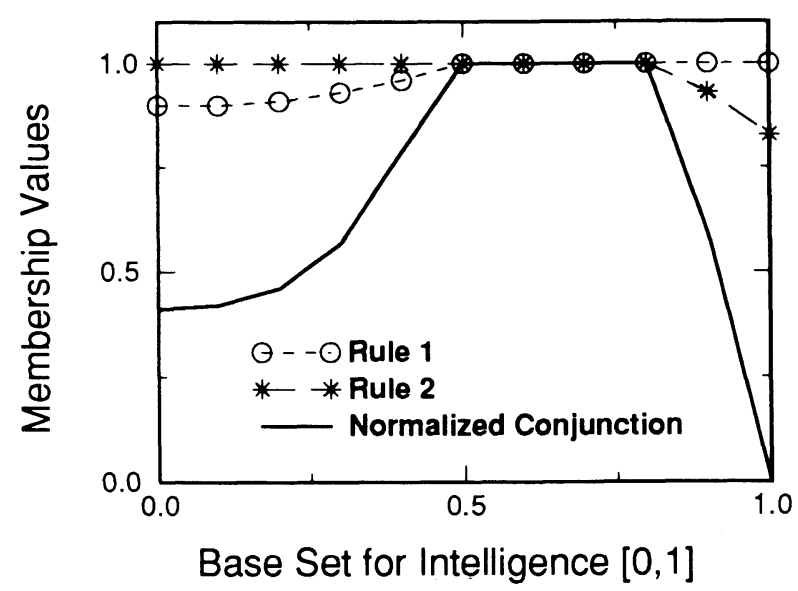

Figure 2. Evaluation membership curves for Rule 1, Rule 2, and their normalized conjunction with intelligence as the referential base set.

Table 5

The Centroid Values for the Fuzzy Category Curves (Figure 1c)

\begin{tabular}{lc}
\hline Fuzzy Category & Centroid Value \\
\hline not & 0.23 \\
somewhat & 0.41 \\
(prototypically) & 0.72 \\
very & 0.76 \\
extremely & 0.79 \\
\hline
\end{tabular}

The next step is to compute the final evaluation. At this juncture, there are different procedures that can be adopted. We use the normalized overall values from Table 4 to construct the curve shown in Figure 2. Comparing this curve against the fuzzy category curves in Figure 1c, we note that it corresponds most closely to the curve for somewhat. The relationship can be seen more clearly if we compare centroid values (Table 5). Therefore, given the raw data in Table 1 and the obtained centroid value of 0.52 , the fuzzy conclusion is that the individual is somewhat intelligent. This forms the output of the fuzzy intelligence evaluation expert system.

\section{Summary and Conclusion}

To summarize, this paper presents a fuzzy intelligence evaluation expert system that performs well in determining the degree of intelligence of an individual. Fuzzy logic and approximate reasoning allow us to consolidate the various sources that contribute to the intelligence of an individual. Moreover, our model can provide group evaluation. In this paper, we illustrated the system using a specific set of membership functions and a minimal rule base. We are in the process of expanding the system and obtaining empirical results to determine the most appropriate membership functions, a more adequate rule base, and an appropriate mechanism for group evaluation.

\section{REFERENCES}

Bellman, R. E., \& ZaDeh, L. A. (1970). Decision making in a fuzzy environment. Management Science, 17, 141-164.

Hinton, G. E. (1989). Learning distributed representations of concepts. In R. G. M. Morris (Ed.), Parallel distributed processing: Implications for psychology and neurobiology (pp. 46-61). Oxford: Clarendon.

Iran-NejaD, A., MarSh, G. E., \& Clements, A. D. (1992). The figure and the ground of constructive brain functioning: Beyond explicit memory processes. Educational Psychologist, 27, 473-492.

Iran-NeJAD, A., \& OrToNy, A. (1984). A biofunctional model of distributed mental content, mental structures, awareness, and attention. Journal of Mind \& Behavior, 5, 173-210.

MARSH, G. E., II, \& IRAN-NEJAD, A. (1992). Intelligence: Beyond a monolithic concept. Bulletin of the Psychonomic Society, 30, 329-332.

Spearman, C. (1927). The abilities of man. New York: Mcmillan.

Wittgenstein, L. (1953). Philosophical investigations. New York: Macmillan.

ZADEH, L. A. (1965). Fuzzy sets. Information Control, 8, 338-353.

ZADEH, L. A. (1977). A theory of approximate reasoning (Memorandum No. UCB/ERL M 77/58). Berkeley: University of California.

(Manuscript received November 16, 1992.) 